\title{
Nano-oxidation of silicon surfaces: Comparison of noncontact and contact atomic-force microscopy methods
}

\author{
Marta Tello and Ricardo García ${ }^{a}$ \\ Instituto de Microelectrónica de Madrid, CSIC, C/Isaac Newton 8, 28760 Tres Cantos, Madrid, Spain
}

(Received 26 February 2001; accepted for publication 23 May 2001)

\begin{abstract}
Local oxidation lithography by atomic-force microscopy is emerging as a powerful method for nanometer-scale patterning of surfaces. Here, we perform a comparative study of contact and noncontact atomic-force microscopy (AFM) oxidation experiments. The comparison of height and width dependencies on voltage and pulse duration allows establishing noncontact AFM as the optimum local oxidation method. For the same electrical conditions, noncontact AFM oxides exhibit higher aspect ratios (0.04 vs 0.02). The smallness of the liquid meniscus in noncontact AFM oxidation produces smaller oxide widths. We also report a slower oxidation rate in contact AFM oxidation. We explain this result by introducing an effective energy barrier $(\sim 0.14 \mathrm{eV})$ that includes the mechanical work done by the growing oxide against the cantilever $(\sim 0.01 \mathrm{eV})$. (c) 2001 American Institute of Physics. [DOI: 10.1063/1.1385582]
\end{abstract}

Nano-oxidation, or local oxidation of semiconductor and metallic surfaces by atomic-force microscopy (AFM), is emerging as a reliable and versatile lithography method for the fabrication of nanometer-scale structures and devices. ${ }^{1-7}$ The strong activity devoted to this lithography technique has revealed some of the relevant factors affecting the oxidation process, such as the voltage and pulse duration, ${ }^{8-10}$ doping of the substrate, ${ }^{11,12}$ formation of a liquid bridge, ${ }^{4,13,14}$ or the chemical composition of the atmosphere. ${ }^{15}$ The present knowledge allows establishing some similarities to conventional anodic oxidation. The AFM tip is used as a cathode and the water meniscus formed between tip and surface is the electrolyte. The strong localization of the electrical field lines near the tip apex gives rise to a nanometer-size oxide dot. However, many details of the oxidation mechanism and kinetics are still under study. ${ }^{9,10,16-18}$

Several AFM modes, such as contact (c-AFM), ${ }^{19}$ tapping mode (intermittent contact), ${ }^{20}$ or noncontact (nc-AFM), ${ }^{21}$ have been used to perform local oxidation lithography. Contact AFM operation is the most extended mode for local oxidation. However, contact forces are responsible for the wear of the tip. To extend the tip's lifetime during the oxidation process and improve the reproducibility of the lithography we have proposed the use of an AFM operated in noncontact mode. ${ }^{4,21}$ It was also suggested that noncontact AFM oxidation produced inherently smaller features (in the lateral dimension) due to the control and smallness of the liquid meniscus. ${ }^{4}$ However, no direct comparisons between local AFM oxidation methods have been provided. Tappingmode AFM could be considered a hybrid of contact and noncontact modes, ${ }^{22}$ so its performance for local oxidation could be inferred from the fundamental AFM modes.

To shed more light onto the mechanism and factors that control the growth of local oxides, we have performed a comparative study of the height and width dependencies on

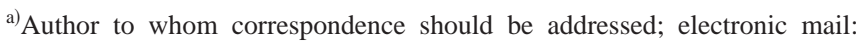
rgarcia@imm.cnm.csic.es
}

voltage pulse conditions in contact and noncontact AFM oxidation.

The experiments were performed with an atomic-force microscope (Nanoscope III, Digital Instruments) with additional circuits to perform the oxidation. The microscope was placed into a closed box with inlets for dry and $\mathrm{H}_{2} \mathrm{O}$ saturated nitrogen. The relative humidity was kept around 30\%$40 \%$. Noncontact AFM oxidations were performed with doped $n^{+}$-type silicon cantilevers ${ }^{23}$ (Nanosensors, Germany). The force constant $\left(k_{c}\right)$ and resonance frequency $\left(f_{0}\right)$ were about $30 \mathrm{~N} / \mathrm{m}$ and $314 \mathrm{kHz}$, respectively. The cantilever was excited at its resonance frequency. Contact AFM experiments were performed with $\mathrm{Si}_{3} \mathrm{~N}_{4}$ cantilevers (Olympus, Japan) with $k_{c} \sim 0.75 \mathrm{~N} / \mathrm{m}$. The $\mathrm{Si}_{3} \mathrm{~N}_{4}$ cantilevers were metallized with a layer $5 \mathrm{~nm}$ of $\mathrm{Cr}$ and $10 \mathrm{~nm}$ of Au. The oxidation was performed by applying a constant force of $1 \mathrm{nN}$. The feedback is switched off during the local oxidation process in both contact and nc-AFM operations. The semiconductor samples were $p$-type $\mathrm{Si}(100)$ with a resistivity of $14 \Omega \mathrm{cm}$. To minimize tip convolution artifacts due to the use of different tips, the measurements have been performed with the same tip. This implies that after a local oxidation experiment, the sample was removed from the oxidation stage to the imaging stage. The numerical data presented in Figs. 2-4 are the average of five oxidation experiments under the same conditions (the standard deviation is approximately $10 \%$ of the average value).

The lateral dimensions of a local oxide depend on several factors, such as the voltage and pulse duration, the relative humidity, the hidrophobicity and dielectric constant of the material to be oxidized, and the tip's size and geometry. To perform a comparison between both methods we have kept unchanged the sample properties, the relative humidity, and the electrical parameters. Ideally, the same tip should be used in both local oxidation methods. However, nc-AFM cantilevers are not suitable for c-AFM operation due to the high values of the force constants used in nc-AFM. To guarantee the use of tips with similar curvature radius, contact and noncontact tips were tested in a calibration sample 


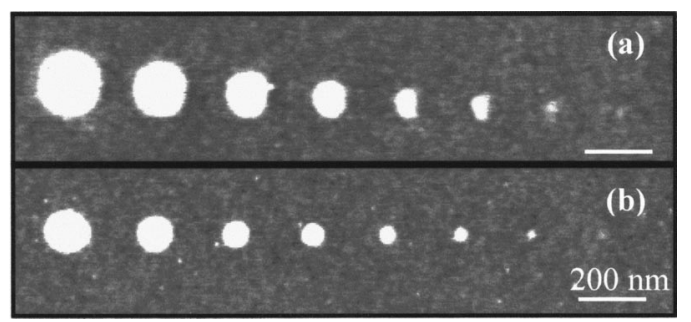

FIG. 1. AFM image of a sequence of oxidation experiments performed for different pulse duration $(3,1,0.3,0.1,0.03,0.01,0.003$, and $0.001 \mathrm{~s}$, from left to right) and constant voltage $(20 \mathrm{~V})$. (a) Contact AFM oxidation; (b) nc-AFM oxidation; $\mathrm{RH}=36 \%$; and horizontal bar, $200 \mathrm{~nm}$.

[10-20 nm InAs quantum dots (QDs) on GaAs(001)]. Only tips that produced similar apparent images of QDs were selected for performing local oxidation experiments.

Figure 1 shows a sequence of oxide dots obtained by contact (a) and nc-AFM operation (b). The dots have been obtained by applying a voltage pulse of $20 \mathrm{~V}$ (tip negative) for different times $(3,1,0.3,0.1,0.03,0.01,0.003$, and 0.001 s). A visual inspection reveals that nc-AFM oxides are smaller than contact oxides.

Figure 2 shows the apparent width dependence for contact and nc-AFM on pulse duration for two voltage pulses. Independently of the oxidation method, the width shows a quasilogarithmic dependence on pulse duration. In all cases, the apparent width is significantly smaller in nc-AFM oxidation. For long pulses $(\geqslant 1 \mathrm{~s})$ the relative difference is about $50 \%$.

It is tempting to attribute the observed differences to the size of the water meniscus. In most experimental conditions, the local oxidation requires the presence of a water meniscus between tip and sample. This meniscus provides the oxyanions $\left(\mathrm{OH}^{-}, \mathrm{O}^{-}\right)$needed to grow the oxide. The meniscus is formed spontaneously in contact operation due to the condensation of water vapor in the nanometer-size cavities of the tip-surface interface. ${ }^{24}$ In contrast, in nc-AFM oxidation the liquid bridge is field induced by the application of an external voltage. ${ }^{4}$ In contact oxidation the operator has little control on the meniscus size. This depends on the geometry

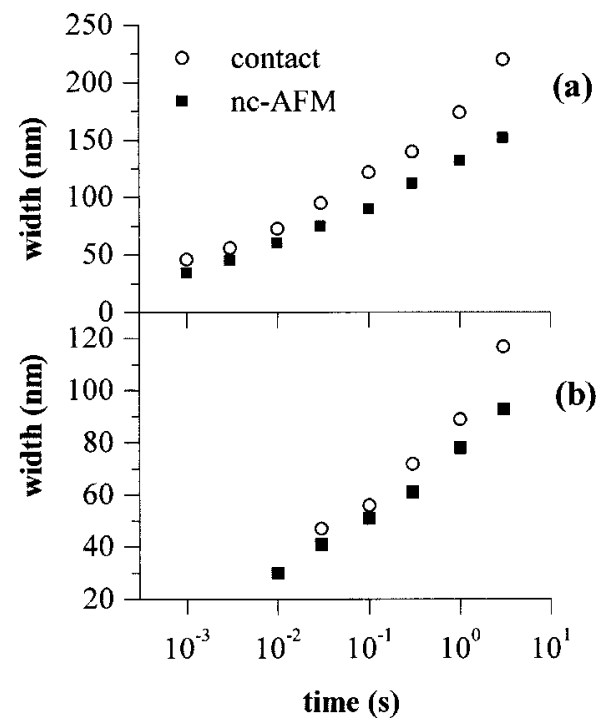

FIG. 2. Apparent dot width dependence on pulse duration and local oxida-

tion method. (a) $V=20 \mathrm{v}$; (b) $V=14 \mathrm{~V}$; and $\mathrm{RH}=36 \%$. deflect the cantilever the oxide's height:
Downloaded 19 Feb 2010 to 161.111 .180 .191 . Redistribution subject to AIP license or copyright; see http://aplaip.or

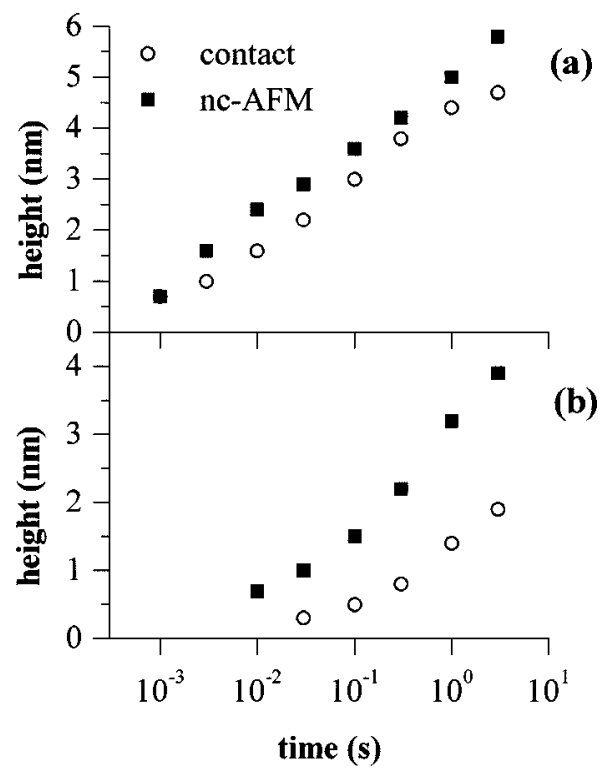

FIG. 3. Dot height dependence on pulse duration and local oxidation method. (a) $V=20 \mathrm{~V}$; (b) $V=14 \mathrm{~V}$; and $\mathrm{RH}=36 \%$.

of the tip-sample interface and the relative humidity. On the contrary, in nc-AFM oxidation, once the liquid bridge has been formed, its lateral dimensions can be decreased by increasing the average tip-sample separation. ${ }^{4}$ The above results show that for similar tip radius, smaller water meniscus can be obtained in nc-AFM.

Figure 3 shows the dot's height dependence on the pulse duration for contact and nc-AFM oxidation methods. There are three main observations. The kinetics of the oxidation seems independent of the oxidation method. It follows an apparent logarithmic law. For the same pulse conditions, the height is considerably higher in noncontact oxidation. This implies that the oxidation rate depends on the AFM oxidation method. This is a rather unexpected result. Contact and noncontact AFM methods differ in the size of the water meniscus and in the tip-sample separation. Those factors do not seem to imply differences in the vertical growth rate. Additionally, for a fixed time the height difference decreases with the applied voltage.

To understand the above observations we need to consider a model of the oxide kinetics. By applying spacecharge-limited growth considerations an expression for the oxide growth rate has been derived: ${ }^{9}$

$$
\frac{d h}{d t} \propto \exp \left(\frac{-W^{\mathrm{eff}}}{k_{B} T}\right) F(h)
$$

where $W^{\text {eff }}$ is related to the energy barrier that an ion has to overcome to move to the next interstitial site, and $F(h)$ includes the terms that contain an explicit dependence with the thickness. The data of Fig. 3 imply that $W_{\mathrm{nc}}^{\text {eff }}<W_{c}^{\text {eff }}$. Specifically, for $V=14 \mathrm{~V}$ effective diffusion barriers of 0.14 and $0.13 \mathrm{eV}$ are obtained for contact and noncontact AFM oxidation, respectively. In c-AFM oxidation, besides the diffusion barrier deduced from the interatomic potential, an effective mechanical barrier due to the cantilever deflection should also be taken into account. This effective barrier can be calculated by considering the mechanical work needed to deflect the cantilever the oxide's height: 


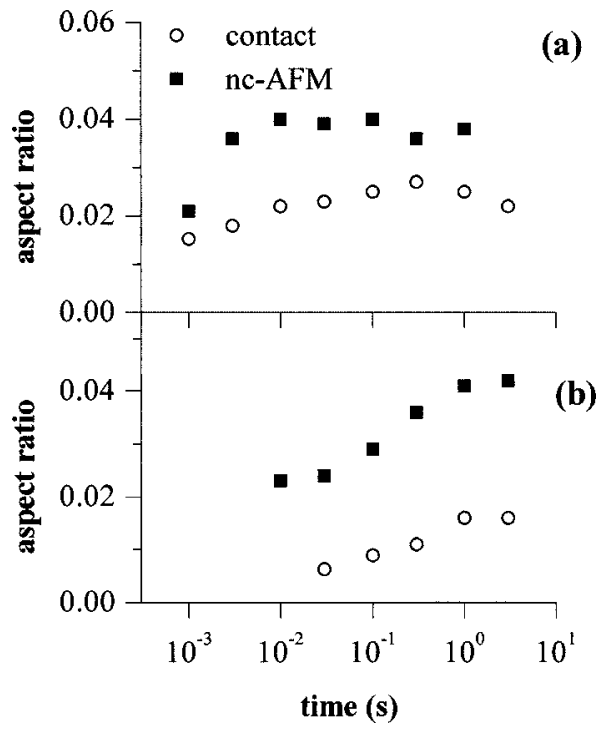

FIG. 4. Aspect ratio dependence on pulse duration and local oxidation method. (a) $V=20 \mathrm{~V}$; (b) $V=14 \mathrm{~V}$; and $\mathrm{RH}=36 \%$.

$$
W_{m}^{\mathrm{eff}}=\frac{\int_{z_{i}}^{z_{f}} k_{c} z d z}{S N}=\frac{k_{c}\left(h^{2}+2 z_{i} h\right)}{2 S N},
$$

where $S$ is the tip-sample contact area divided by the unit area of $\mathrm{SiO}_{2}\left(0.12 \mathrm{~nm}^{2}\right)$ and $N$ is the number of $\mathrm{SiO}_{2}$ unit cells along the $z$ direction; $h$ is the height of the oxide, and $z_{i}$ the initial deflection of the cantilever. For $V=14 \mathrm{~V}$ and $t=3 \mathrm{~s}$, Eq. (2) gives $W_{m}^{\mathrm{eff}} \approx 0.02 \mathrm{eV}$. This value is quite close to the difference found between $W_{\mathrm{nc}}^{\mathrm{eff}}$ and $W_{c}^{\mathrm{eff}}(0.01 \mathrm{eV})$. In the calculation we have assumed a contact area of $100 \mathrm{~nm}^{2}$ and $N$ $=9$.

Figure 3 also shows a decreasing of $h^{\mathrm{nc}}-h^{c}$ when the applied voltage is increased. This observation can also be explained by the mechanical barrier model. For a fixed time, increasing the voltage implies a large oxide dot (in height and width). This, in turn, decreases the effective mechanical barrier per bond or unit cell. As a consequence, the differences between noncontact and contact AFM oxidations should decrease.

Plotting the aspect ratio of the dots summarizes the results shown in Figs. 2 and 3. Figure 4 show the aspect ratio as a function of the pulse duration for both oxidation methods. Independently of the pulse duration and applied voltage, the aspect ratio is always higher in nc-AFM oxidation.

In summary, we have compared local oxidation experiments performed in contact and nc-AFM modes. Noncontact AFM oxidation produces higher aspect ratios. This observation is a consequence of two effects. First, nc-AFM allows controlling the lateral size of the liquid meniscus. This, in turn, controls the lateral size of the oxide dot. Second, the vertical growth rate is smaller in contact AFM oxidation. We propose that this is a consequence of the mechanical energy needed to deflect the cantilever during the growth of the oxide. The above results lead us to recommend the use of an AFM operated in noncontact mode for performing local oxidation lithography.

The authors acknowledge fruitful discussions with Monserrat Calleja. This work was financially supported by the Dirección General de Enseñanza Superior e Investigación (PB98-0471) and the European Commission (GR5D-CT2000-00349).

${ }^{1}$ E. S. Snow, P. M. Campbell, F. A. Buot, D. Park, C. R. K. Marrian, and R. Magno, Appl. Phys. Lett. 72, 3071 (1998).

${ }^{2}$ S. C. Minne, J .D. Adams, G. Yaralioglu, S. R. Manalis, A. Atalar, and C. F. Quate, Appl. Phys. Lett. 73, 1742 (1998).

${ }^{3}$ R. Held, T. Vancura, T. Heinzel, K. Ensslin, M. Holland, and W. Wegscheider, Appl. Phys. Lett. 73, 262 (1998).

${ }^{4}$ R. García, M. Calleja, and H. Rohrer, J. Appl. Phys. 86, 1898 (1999).

${ }^{5}$ F. S. Chien, C.-L. Wu, Y.-C. Chou, T. T. Chen, S. Gwo, and W.-F. Hsieh, Appl. Phys. Lett. 75, 2429 (1999).

${ }^{6}$ K. Matsumoto, Y. Gotoh, T. Maeda, J. A. Dagata, and J. S. Harris, Appl. Phys. Lett. 76, 239 (2000).

${ }^{7}$ R. Maoz, E. Frydman, S. R. Cohen, and J. Sagiv, Adv. Mater. 12, 725 (2000).

${ }^{8}$ P. Avouris, T. Hertel, and R. Martel, Appl. Phys. Lett. 71, 285 (1997).

${ }^{9}$ E. Dubois and J.-L. Bubendorff, J. Appl. Phys. 87, 8148 (2000).

${ }^{10}$ M. Calleja and R. García, Appl. Phys. Lett. 76, 3427 (2000).

${ }^{11}$ J. A. Dagata, T. Inoue, J. Itoh, K. Matsumoto, and H. Yokoyama, J. Appl. Phys. 84, 6891 (1998).

${ }^{12}$ T. Teuschler, K. Mahr, S. Miyazaki, M. Hundhausen, and L. Levy, Appl. Phys. Lett. 67, 3144 (1995)

${ }^{13}$ H. Sugimura and N. Nakagiri, Jpn. J. Appl. Phys., Part 1 34, 3406 (1995).

${ }^{14}$ K. Morimoto, K. Araki, K. Yamashita, K. Motira, and M. Niwa, Appl. Surf. Sci. 117, 652 (1997).

${ }^{15}$ F. Marchi, V. Bouchiat, H. Dallaporta, V. Safarov, D. Tonneau, and P. Doppelt, J. Vac. Sci. Technol. B 16, 1 (1998).

${ }^{16}$ J. A. Dagata, F. Pérez-Murano, G. Abadal, K. Morimoto, T. Inoue, J. Itoh, and H. Yokoyama, Appl. Phys. Lett. 76, 2710 (2000).

${ }^{17}$ Y. Y. Wei and G. Eres, Appl. Phys. Lett. 76, 194 (2000).

${ }^{18}$ F. S. S. Chien, Y.-C. Chou, T. T. Chen, W.-F. Hsieh, T. S. Chao, and S. Gwo, J. Appl. Phys. 89, 2465 (2001).

${ }^{19}$ H. C. Day and D. R. Allee, Appl. Phys. Lett. 62, 2691 (1993).

${ }^{20}$ F. Pérez-Murano, G. Abadal, N. Barniol, X. Aymerich, J. Servat, P. Gorostiza, and F. Sanz, J. Appl. Phys. 78, 6797 (1995).

${ }^{21}$ R. García, M. Calleja, and F. Pérez-Murano, Appl. Phys. Lett. 72, 2295 (1998).

${ }^{22}$ R. García, and A. San Paulo, Phys. Rev. B 60, 4961 (1999).

${ }^{23}$ Gold-coated silicon tips were also used to perform noncontact AFM oxidation experiments. The growth rate shows a slight dependence on the tip composition. However, the conclusions presented here are independent of the composition of the tip.

${ }^{24}$ J. Israelachvili, Intermolecular and Surface Forces (Academic, London, 1992). 